\title{
Effect of a 12-week training program on the anthropometric and physiological profiles of some participants registered in a fitness center in Douala, Cameroon
}

\author{
Peguy Brice Assomo Ndemba ${ }^{1,2}$, Clarisse Noël Ayina Ayina', Wiliam Richard Guessogo ${ }^{3}$, Jerson Mekoulou Ndongo', \\ Rita Ornella Ghontchouang Kamnang', Claude Elysée Bika Lele', Samuel Honoré Mandengue ${ }^{1,4}$, Laurent Serge Etoundi-Ngoa², \\ Abdou Temfemo ${ }^{1,4, *}$ \\ ${ }^{1}$ Exercise and Sport Physiology Unit, Faculty of Sciences, University of Douala, Douala, Cameroon \\ 2Department of Physiology, Faculty of Medicine and Biomedical Sciences, University of Yaounde I, Yaounde, Cameroon \\ ${ }^{3}$ National Institute of Youth and Sports of Yaounde, Yaounde, Cameroon \\ ${ }^{4}$ Department of Biological Sciences, Faculty of Medicine and Pharmaceutical Sciences, University of Douala, Douala, Cameroon
}

Fitness centers are remarkably abundant in Cameroon. The aim of this work was to assess the effects of a 12-week training program on the anthropometric and physiological profiles of some participants in a fitness center. A total of 86 participants ( 40 from the experimental group and 46 from the control group) with age ranging from 17 to 53 years were subjected to pre- and posttraining assessments of, anthropometric parameters, physiological parameters, and performance. Anthropometric parameters (weight, height, body mass index [BMI], waist circumference [WC]) and blood pressure (diastolic blood pressure, systolic blood pressure [SBP]) were measured according to standard protocols. Heart rate was recorded using a heart rate monitor. Cardiorespiratory fitness (maximal oxygen uptake or $\mathrm{VO}_{2 \text { peak }}$ ) was estimated by the 20-multistage shuttle run test. All the data was collected twice within 12 weeks. Weight, BMI, and WC did not show any significant variation $(P>0.05)$ after a 12-week training program. $\mathrm{VO}_{2 \text { peak }}$ increase was insignificant $(P>0.05)$ higher in men $(7.5 \%, P=0.06)$, compared to women $(5.4 \%, P=0.4)$. We noticed a significant reduction $(P=0.002)$ in the SBP of men. Significant increase of $\mathrm{HR}$ max was found in women. There was an improvement of $13.7 \%$ in the $\mathrm{VO}_{2 \text { peak }}$ of the participants who did not consume alcohol. These results demonstrate the slight benefits of a 12week training program on health. The weight characteristics of the participants and lifestyle may play an important role in these interactions.

Keywords: Training program, Fitness center, Healthy participants, Cameroon

\section{INTRODUCTION}

Physical inactivity is a pandemic over the world. More than $31 \%$ of individuals do not meet the recommendations related to regular physical and sports activities (Kohl et al., 2012). Several factors contribute to this increase in physical inactivity such as increasing urbanization, rapid economic development, rural exodus. Physical inactivity is the fourth leading cause of death worldwide (Kohl et al., 2012). The public policy strategies usually used to deal with this growing phenomenon of physical inactivity involve installing sports infrastructures in different places (Deelen et al., 2018). Commercial sports centers and gymnasiums are also tools for structured participation in physical and sporting activities (Eime et al., 2016; Kamphuis et al., 2008). Some individuals prefer free participation, by investing in public spaces such as sidewalks for walking or running activities (Eime et al., 2016; Kamphuis et al., 2008).

Scientific literature has high interest, to the benefits of training programs in people who present a particular risk (obesity, in people at risk) or a given pathology (Guessogo et al., 2016a; Guesso-
${ }^{*}$ Corresponding author: Abdou Temfemo (iD https://orcid.org/0000-0002-6449-9512 Faculty of Medicine and Pharmaceutical Sciences of the University of Douala, Douala, Cameron; Université de Picardie Jules Verne, Amiens, Cedex, France E-mail: temfemo@hotmail.com

Received: May 12, 2020 / Accepted: June 16, 2020
This is an Open Access article distributed under the terms of the Creative Commons Attribution Non-Commercial License (https://creativecommons.org/licenses/by-nc/4.0/) which permits unrestricted non-commercial use, distribution, and reproduction in any medium, provided the original work is properly cited. 
go et al., 2016b; Park et al., 2019; Sun et al., 2019). The training programs implemented in these studies differ in intensity, duration, and protocols, thus justifying sometimes controversial results.

In Cameroon, health policies focus mainly on the fight against human immunodeficiency virus infection/acquired immune deficiency syndrome, tuberculosis, malaria. However, nontransmissible diseases and some associated risk factors such as overweight and obesity are already growing alarmingly (Choukem et al., 2017; Juma et al., 2018). For instance, some studies conducted in the Cameroonian context revealed alarming prevalences of overweight and obesity in all age groups (Choukem et al., 2017; Navti et al., 2014). Navti et al. (2014) observed an inverse association between body mass index (BMI) and physical activity among Cameroonian school children. Unfortunately, the implementation of the objective, that is the assessment of the physical activity that they have suggested is limited due to the lack of relative studies. However, behaviors such as participation in physical and sports activities in both at-risk and healthy individuals are assimilated and gradually becoming more westernized in the Cameroonian context. It is very common to meet people who practice walking, running, and even cycling in public spaces and the streets. But unlike developed countries where the government invest to set up sports facilities for the public, commercial fitness centers are remarkably abundant. These centers are home to normal, overweight, obese individuals as well as those having particular conditions (diabetes, high blood pressure).

In the study of Guessogo et al. (2016b), an improvement in body composition and lipid profile was observed in obese women after 24 weeks of a training program including $2 \mathrm{~km}$ and 6-min walking tests. However, in the same study, no significant change in these same parameters was observed after 12 weeks. Thus, the nature and structure of training protocols, their duration, the training period are important parameters that need to be considered in optimizing training programs. Owing to the increased adhesion of a heterogeneous population (normal and ill) to fitness centers, it becomes appropriate to assess the effectiveness of the programs offered by these structures. Previous study (Guessogo et al., 2016b) has been conducted in outdoor settings. However, sports participation in outdoor settings can also produce higher restorative health benefits than do indoor settings (Hug et al., 2009). This study aims to assess the anthropometric, physiological, and cardiorespiratory profiles of some participants registered in a fitness center.

\section{MATERIALS AND METHODS}

\section{Participants}

This study involved 86 participants, 40 from the experimental group and 46 from the control group. During the first data acquisition (anthropometric, physiological parameters, and performance), 67 participants from the experimental group were included, but only 40 participated in the second data collection after 12-week month of training. The longitudinal nature of the study and the unavailability of some participants were the main reasons for the decrease in the sample.

This study was conducted from February to June 2018. These experiments were carried out in around at 6 a.m. and at 6 p.m.

Participant's selection was based on some information available in the center's follow-up register, excluding participants with diabetes, high blood pressure, or any other disease. Participants in this center paid a quarterly fee for blood glucose and lipid profile measurements.

The ethical considerations of our study were validated through the ethical clearance issued by the Institutional Ethics Committee of Research for Human Health of the University of Douala under the number 1322 CEI-Udo/11/2017/M. At the same time, letters were sent to some fitness center managers requesting their permissions and collaborations for the achievement of the study's objectives. Only one center showed interest in our study by inviting all its affiliated members to participate in the study, through various types of communication (posting, oral communication) initiated by the head of the center. Participants in the control group were selected based on friendly relationships between them and the experimenters. This group consisted of people who do not perform regular physical activity. The control group did not take part in the training program and continued their daily activities normally. They only took part in two 20-m multistage shuttle run tests over 3 months.

\section{Experimental protocol}

Before the start of the experimental protocol, participants were interviewed by the researchers in simple terms on the different modalities of the 20-m multistage shuttle run test and the objectives of the study. Then, at the request of the experimenters, each participant filled out a card including the following information: names and surnames, age, sports discipline, profession, telephone number. The rest of the information on the experimental data was collected by the experimenters. The experimental protocol involved the performing of the 20-m multistage shuttle run test in which 
physiological (heart rate) and performance (number of shuttles) parameters were measured. Before the test, anthropometric parameters were collected including the measurement of weight, height, and waist circumference. The heart rate was monitored continuously during the 20-m multistage shuttle run test (Polar V800, Polar Electro Oy, Kempele, Finland). The 20-m multistage shutthe run test was used to assess the cardiorespiratory fitness. Participants were requested to run between two lines $20 \mathrm{~m}$ apart at a pace marked by a prerecorded sound signal. The initial speed was $8.5 \mathrm{~km} / \mathrm{hr}$, with $0.5-\mathrm{km} / \mathrm{hr}$ increments each stage. The test was stopped when the participant failed to reach the line twice in a row. The last half-stage attained was recorded. Immediately after the end of the race, the number of bearings was noted for the calculation of the $\mathrm{VO}_{2}$ pic by a regression equation developed by Léger et al. (1988). To recover participants were asked by researchers to sit down. Heart rate, 1-min postexercise (HR1) was read on the heart rate monitor (Polar V800). The maximum heart rate (HRmax) was read, recorded and the difference with the HR1 was used to calculate the heart rate recovery. The same experimental protocol was repeated 3 months later to evaluate the effects of the training program on anthropometric, physiological, and cardiorespiratory parameters

\section{Training program}

All participants in the experimental group were enrolled to follow a training program at a rate of four sessions per week (Fig. 1). For a better follow-up of the regularity of the sessions with the participants, a list of 48 attendances was made available to the instructor at the rate of 4 sessions per week. The effective participation rate in the 4 weekly sessions was $81.25 \%$ or $36 / 48$. The absences observed were linked to illnesses, family occupations, or trips outside the city. Each session lasted $1 \mathrm{hr}$ and consisted of aerobic and anaerobic exercises. These sessions most often began with dances punctuated by music (10 min), followed by stretching (10 $\mathrm{min})$ and muscle building ( $40 \mathrm{~min})$. The exercises were similar during each session. Each session consisting of: (a) a 20-min-long warm-up performed as follows: 5 min of a pedalling exercise on an ergocycle with the hands and feet, 5 min of dance that is to step up and step down between a bench $5 \mathrm{~m}$ high and the floor with each leg and 10 min of respiratory movements. (b) a main exercise was determined from the result of the 20-m multistage shuttle run test. We considered the initial speed $(\mathrm{V} 0=8.5 \mathrm{~km} / \mathrm{hr})$ and the maximum speed or that of the last level (Vmax). To determine the training speed (VE), an intensity of $60 \%$ of the reserve speed $(\mathrm{VR}=\mathrm{Vmax}-\mathrm{V} 0)$ was calculated for each participant and $\mathrm{VE}$ was

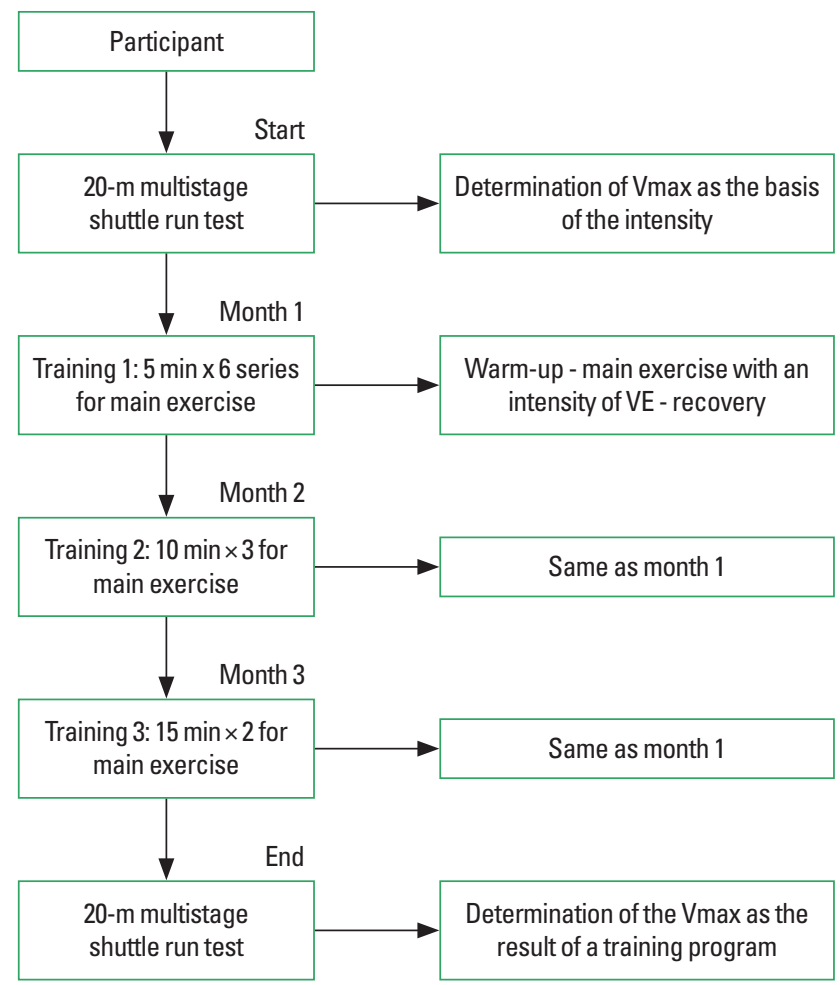

Fig. 1. Experimental procedure.

then equal to V0+60\% VR. The main exercise took place around a 120-m perimeter circuit delimited inside the fitness centre. The time (t120) to complete $120 \mathrm{~m}$ (1tr) was calculated for each participant from VE, with $\mathrm{t} 120=120 \mathrm{~m} / \mathrm{VE}$. Three stages of evolution were selected $(5,10$, and $15 \mathrm{~min}$ in 6,3 , and 2 series of certain number of turn, respectively for the first, second, and third month). The number of turns for each series was equal to $\mathrm{tE} / \mathrm{t} 120$ (with $\mathrm{tE}=$ time to the training). The time to complete each $120 \mathrm{~m}$, as well as the number of laps for 5, 10, and $15 \mathrm{~min}$ and for each subject, were entered in a metronome.

\section{Statistical analysis}

The data obtained during these experiments were recorded in an Excel sheet (version 2013). Depending on normality and homogeneity conditions, the Wilcoxon test was used to perform a comparative analysis of the effects of training programs on anthropometric, physiological, and cardiorespiratory parameters. Each subject was its control. The study of correlations between the different parameters (anthropometry, physiological parameters, and performance) was carried out by calculating the Bravais-Pearson linear correlation coefficient. The data were expressed on average \pm standard deviation for each of the parameters of the studied 
Assomo Ndemba PB, et al. • Biological responses to indoor training program

Table 1. Physiological and anthropometric parameters of participants to 12-week training program

\begin{tabular}{|c|c|c|c|c|c|c|}
\hline \multirow{2}{*}{ Parameter } & \multicolumn{3}{|c|}{$\operatorname{EXP}(n=40)$} & \multicolumn{3}{|c|}{$\operatorname{CON}(n=46)$} \\
\hline & Pre & Post & $P$-value & Pre & Post & $P$-value \\
\hline \multicolumn{7}{|c|}{ Weight (kg) } \\
\hline Male & $76.9 \pm 11.3$ & $76.6 \pm 11.4$ & 0.39 & $75.6 \pm 10.7$ & $75.8 \pm 11.2$ & 0.45 \\
\hline Female & $71.6 \pm 15.3$ & $71.3 \pm 15.2$ & 0.9 & $70.7 \pm 14.8$ & $70.3 \pm 13.9$ & 0.85 \\
\hline \multicolumn{7}{|c|}{ BMI (kg/m²) } \\
\hline Male & $25.5 \pm 4.0$ & $25.4 \pm 4.0$ & 0.48 & $26.3 \pm 3.8$ & $26.7 \pm 4.5$ & 0.62 \\
\hline Female & $26.8 \pm 5.5$ & $26.7 \pm 5.5$ & 0.9 & $27.7 \pm 4.9$ & $27.3 \pm 5.2$ & 0.86 \\
\hline \multicolumn{7}{|c|}{ Waist-hip ratio } \\
\hline Male & $83.7 \pm 8.5$ & $83.0 \pm 8.5$ & 0.27 & $82.5 \pm 9.6$ & $82.8 \pm 8.1$ & 0.35 \\
\hline Female & $84.7 \pm 10.6$ & $84.3 \pm 10.2$ & 0.7 & $83.5 \pm 9.8$ & $83.7 \pm 10.4$ & 0.8 \\
\hline \multicolumn{7}{|l|}{$\mathrm{HRr}$ (bpm) } \\
\hline Male & $89.6 \pm 11$ & $87.5 \pm 17$ & 0.5 & $91.3 \pm 9.8$ & $91.7 \pm 10.5$ & 0.76 \\
\hline Female & $93.5 \pm 18.3$ & $91.4 \pm 17.0$ & 0.38 & $94.9 \pm 16.7$ & $92.2 \pm 16.5$ & 0.82 \\
\hline \multicolumn{7}{|l|}{ HR1 (bpm) } \\
\hline Male & $125.5 \pm 23.7$ & $134.8 \pm 30.9$ & $0.02^{*}$ & $128.5 \pm 22.8$ & $128.2 \pm 23.6$ & 0.65 \\
\hline Female & $137.5 \pm 40.9$ & $139.0 \pm 35.8$ & 0.6 & $139.2 \pm 35.7$ & $139.6 \pm 35.9$ & 0.58 \\
\hline \multicolumn{7}{|c|}{ HRmax (bpm) } \\
\hline Male & $180.4 \pm 17.8$ & $183.0 \pm 15.0$ & 0.14 & $183.5 \pm 15.8$ & $184.9 \pm 22.2$ & 0.74 \\
\hline Female & $182.5 \pm 23.4$ & $191.3 \pm 22.6$ & $0.0052^{*}$ & $185.6 \pm 21.4$ & $184.9 \pm 22.2$ & 0.69 \\
\hline \multicolumn{7}{|c|}{$\mathrm{DBP}(\mathrm{mmHg})$} \\
\hline Male & $78.6 \pm 9.7$ & $76.8 \pm 6.9$ & 0.69 & $80.3 \pm 8.8$ & $80.7 \pm 7.7$ & 0.45 \\
\hline Female & $83.1 \pm 15.2$ & $77.3 \pm 12.6$ & 0.09 & $82.6 \pm 13.5$ & $82.1 \pm 12.9$ & 0.57 \\
\hline \multicolumn{7}{|c|}{$\mathrm{SBP}(\mathrm{mmHg})$} \\
\hline Male & $128.1 \pm 11.4$ & $116.0 \pm 22.7$ & $0.002^{*}$ & $130.4 \pm 10.7$ & $129.5 \pm 10.5$ & 0.73 \\
\hline Female & $119.1 \pm 19.2$ & $117.2 \pm 21.9$ & 0.47 & $117.2 \pm 17.3$ & $116.9 \pm 16.8$ & 0.61 \\
\hline \multicolumn{7}{|c|}{$\mathrm{VO}_{2 \text { peak }}(\mathrm{mL} / \mathrm{kg} / \mathrm{min})$} \\
\hline Male & $42.6 \pm 6.7$ & $45.8 \pm 9.1$ & 0.06 & $39.5 \pm 7.2$ & $39.8 \pm 6.5$ & 0.70 \\
\hline Female & $29.9 \pm 7.0$ & $31.5 \pm 6.8$ & 0.4 & $25.7 \pm 6.8$ & $25.3 \pm 6.3$ & 0.65 \\
\hline
\end{tabular}

Values are presented as mean \pm standard deviation.

EXP, experimental group; CON, control group; Pre, before the training period; Post, after 12 weeks of training program; BMl, body mass index; HRr, resting heart rate; HR1, heart rate one minute after recovery; HRmax, maximum heart rate; DBP, diastolic blood pressure; SBP, systolic blood pressure; $\mathrm{VO}_{2 p e a k}$, peak of oxygen consumption.

${ }^{*} P<0.05$, difference between male and female.

population. The differences were considered statistically significant for $P<0.05$.

\section{RESULTS}

Table 1 shows the variation in anthropometric parameters (height, weight, BMI, and waist circumference) in response to a 12-week training program. After 12 weeks of training, the weight, BMI, and waist circumference did not show significant changes in both men and women $(P>0.05)$. Similar results were observed in the control group. Concerning blood pressure parameters, a significant decrease was observed in systolic blood pressure (SBP) of men ( $P=$ $0.002)$ from the experimental group. Also, there was a significant increase in the HR1 $(P=0.02)$ in men and the HRmax $(P=0.005)$ in women, after 12-week training program. The $\mathrm{VO}_{\text {2peak }}$ of men showed more improvement $(P=0.06)$ as compared to women $(P=$ 0.4 ) in the experimental group (Table 1).

Our sample initially included $45 \%$ of normal-weight individuals. After 3 months of training, we noticed an increase in the prevalence of obesity in men. On the contrary, the prevalence of overweight participants decreased. However, both overweight and obesity prevalence's were stable among women. Moreover, the prevalence of obesity was higher in women compared to men (Table 2). HR1 and HRmax significantly increased in the group eating twice a day (Table 3). $\mathrm{VO}_{2 \text { peak }}$ values improved significantly in the alcohol-free group $(P=0.045)$ and in the group that rarely consumed 
Table 2. Changes of weight status in the experimental group after 12-week training program $(n=40)$

\begin{tabular}{|c|c|c|c|c|c|c|}
\hline \multirow{2}{*}{$\mathrm{BMI}\left(\mathrm{kg} / \mathrm{m}^{2}\right)$} & \multicolumn{3}{|c|}{ Pre } & \multicolumn{3}{|c|}{ Post } \\
\hline & Male & Woman & Total & Male & Woman & Total \\
\hline Normal weight (18.5-24.9) & $11(47.8)$ & $7(41.2)$ & $18(45.0)$ & $12(52.2)$ & $7(41.2)$ & $19(47.5)$ \\
\hline Overweight (25-29.9) & $9(39.1)$ & $6(35.3)$ & $15(37.5)$ & $7(30.4)$ & $6(35.3)$ & $13(32.5)$ \\
\hline Obesity (type 1) (30-34.9) & $2(8.7)$ & $3(17.6)$ & $5(12.5)$ & $4(17.5)$ & $3(17.6)$ & $7(17.5)$ \\
\hline Obesity (type 2) (35-39.9) & $1(4.3)$ & $1(5.9)$ & $2(5.0)$ & - & $1(5.9)$ & $1(2.5)$ \\
\hline
\end{tabular}

Values are presented as number (\%).

BMl, body mass index; Pre, before the training period; Post, after 12 weeks of training program.

Table 3. Effects of the training program on physiological and anthropometric parameters according to the number of meals per day (experimental group, $n=40$ )

\begin{tabular}{|c|c|c|c|c|c|c|}
\hline \multirow{2}{*}{ Parameter } & \multicolumn{3}{|c|}{2 Meals per day $(n=23)$} & \multicolumn{3}{|c|}{3 Meals per day $(n=17)$} \\
\hline & Pre & Post & $P$-value & Pre & Post & $P$-value \\
\hline $\mathrm{BMI}\left(\mathrm{kg} / \mathrm{m}^{2}\right)$ & $26.9 \pm 4.9$ & $26.6 \pm 4.6$ & 0.4 & $25.1 \pm 4.4$ & $25.1 \pm 4.6$ & 0.9 \\
\hline HRr (bpm) & $89.1 \pm 13.5$ & $90.4 \pm 18.6$ & 0.6 & $93.8 \pm 15.6$ & $92.3 \pm 16.2$ & 0.6 \\
\hline HR1 (bpm) & $123.4 \pm 24.5$ & $131.3 \pm 26.4$ & $0.01^{*}$ & $139.3 \pm 38.7$ & $143.3 \pm 38.7$ & 0.9 \\
\hline HRmax (bpm) & $178.0 \pm 15.0$ & $184.9 \pm 13.6$ & $0.047^{*}$ & $185.3 \pm 24.8$ & $188.6 \pm 23.9$ & 0.13 \\
\hline $\mathrm{VO}_{2 \text { peak }}(\mathrm{mL} / \mathrm{kg} / \mathrm{min})$ & $37.6 \pm 9.7$ & $41.5 \pm 11.6$ & 0.06 & $36.8 \pm 8.9$ & $37.6 \pm 9.7$ & 0.4 \\
\hline
\end{tabular}

Values are presented as mean \pm standard deviation.

Pre, before the training period; Post, after 12 weeks of training program; BMI, body mass index; HRr, resting heart rate; HR1, heart rate one minute after recovery; HRmax, maximum heart rate; $\mathrm{VO}_{2 \text { peak, }}$ peak of oxygen consumption.

${ }^{*}$ Difference between pre and post, $P<0.05$.

Table 4. Effects of the training program on physiological and anthropometric parameters according to alcohol consumption (experimental group, $\mathrm{n}=40$ )

\begin{tabular}{|c|c|c|c|c|c|c|c|c|c|}
\hline \multirow{2}{*}{ Parameter } & \multicolumn{3}{|c|}{ Alcohol (none) $(n=12)$} & \multicolumn{3}{|c|}{ Alcohol (moderately) $(\mathrm{n}=9)$} & \multicolumn{3}{|c|}{ Alcohol (rarely) $(n=19)$} \\
\hline & Pre & Post & $P$-value & Pre & Post & $P$-value & Pre & Post & $P$-value \\
\hline $\mathrm{BMI}\left(\mathrm{kg} / \mathrm{m}^{2}\right)$ & $25.4 \pm 5.5$ & $25.0 \pm 3.2$ & 0.33 & $28.7 \pm 5.5$ & $28.6 \pm 5.3$ & 0.7 & $24.8 \pm 4.2$ & $24.8 \pm 4.3$ & 1 \\
\hline $\mathrm{HRr}$ (bpm) & $89.5 \pm 14.2$ & $82.4 \pm 13.5$ & 0.3 & $89.4 \pm 8.6$ & $90.9 \pm 18.5$ & 0.8 & $93.0 \pm 17.5$ & $95.0 \pm 17.4$ & 0.6 \\
\hline HR1 (bpm) & $127.9 \pm 40.9$ & $139.0 \pm 47.3$ & 0.3 & $129.9 \pm 31.4$ & $134.4 \pm 33.2$ & 0.07 & $132.0 \pm 30.6$ & $136.9 \pm 26.7$ & 0.2 \\
\hline HRmax (bpm) & $186.5 \pm 24.0$ & $187.6 \pm 15.9$ & 0.7 & $180.4 \pm 15.0$ & $188.2 \pm 18.0$ & 0.045 & $179.7 \pm 21.7$ & $185.2 \pm 20,9$ & 0.02 \\
\hline $\mathrm{VO}_{\text {2peak }}(\mathrm{mL} / \mathrm{kg} / \mathrm{min})$ & $38.6 \pm 5.8$ & $43.9 \pm 8.7$ & $0.045^{*}$ & $37.9 \pm 10.7$ & $35.9 \pm 13.1$ & 0.5 & $36.4 \pm 9.8$ & $40.4 \pm 9.7$ & 0.02 \\
\hline
\end{tabular}

Pre, before the training period; Post, after 12 weeks of training program; BMl, body mass index; HRr, resting heart rate; HR1, heart rate one minute after recovery; HRmax, maximum heart rate; $\mathrm{DBP}$, diastolic blood pressure; $\mathrm{SBP}$, systolic blood pressure; $\mathrm{VO}_{2 \text { peak, }}$ peak of oxygen consumption.

${ }^{*} P<0.05$, difference between pre and post.

alcohol $(P=0.02)$. A significant increase in HRmax was observed in groups that rarely $(P=0.02)$ and moderately $(P=0.045)$ consumed of alcohol (Table 4).

\section{DISCUSSION}

The main objective of this study was to assess the effect of a 12week training program on the anthropometric and physiological profiles of some participants registered in a fitness center in Douala, Cameroon. This study revealed that after 12 weeks of training, the anthropometric data recorded (weight, height, BMI, and waist circumference) did not change in both sexes. These results are con- sistent with those of Guessogo et al. (2016b). Indeed, these authors studied the effects of a 24-week period of regular physical and sports activities on anthropometric and lipid profiles in obese or overweight women in Cameroon. The results did not show significant differences in weight and BMI after 12 weeks. Our results suggested that a 12-week training program appears insufficient to observe changes in anthropometric parameters in participants. Wong et al. (2008) argued that it takes more than 3 months to record physical benefits inherent in regular physical and sports activities. Guessogo et al. (2016b) assessed the impact of a 24-week training program including $6 \mathrm{~min}$ and 2-km walking tests on anthropometric and lipid profiles in a population of obese Cameroonian women; 
improvements (anthropometric and lipid) were observed after 24 weeks and not after 12 weeks. This time-dependent data on the improvement of biological parameters can evolve especially when taking into consideration nature, duration, and intensity. Several studies have reported the benefits of regular physical and sports activities for times of 12 weeks or less (Brisebois et al., 2018; Murawska-Cialowicz et al., 2015; Park et al., 2019; Posnakidis et al., 2020; Sun et al., 2019). These studies, which are mostly Caucasian, use a high-intensity functional training (HIFT) program. Thus, the high intensity that characterizes this type of training allows having significant results after a few weeks. This shows that the intensities of the exercises used in the fitness center of this study were low. It is likely the reason why the study of Guessogo et al. (2016b) showed significant improvements in body and lipid parameters which were achieved after 24 weeks of a training program including $2 \mathrm{~km}$ and 6-min walking tests. Another factor that needs to be taken into consideration is the qualification of the people who conduct the training in these centers. These centers mostly use available people who are passionate about the sport without appropriate scientific knowledge in the field of physical and sports activities. This may justify the monotony of the exercises that were performed at each session in this center as well as the use of nonage dependent exercise. For instance, our sample included young and old participants who were required to perform the same types of exercises and forces to follow the pace imposed by the instructor.

On the staturo-weight level, this study revealed that after 3 months of training, there was an increase in the prevalence of general obesity in men while the prevalence of abdominal obesity was absent. This result could be explained by an increase in muscle mass. A consistent prevalence of obesity among women was observed between the two periods of the study. Overweight, which is a precursor to obesity was also observed in significant proportions in both men and women. The prevalence of overweight (37.5\%) among men and women observed in this study is similar to that of Schroeder et al. (2017) which reported a prevalence of 37\% overweight among participants in a health club. By considering waist circumference, the prevalence of abdominal obesity in women had increased after 3 months of training. This prevalence is much higher than that of Schroeder et al. (2017) which had reported a prevalence of $20 \%$ among female members in a physical activity club.

The importance of the participants' weight characteristics should be noted in the benefits observed during training programs. The size of our participants had a relatively normal trend with a low rate of obese people. The dominance of normal weight and low-risk participants (overweight) enrolled in this center could explain the relative stabilization of the parameters observed after 3 months of a training program. Thus, these participants with weight characteristics considered as good health would not be quick to adhere to the high intensities of the exercises proposed during the various sessions. The goal is to maintain their fitness; significant improvements in response to training programs of the same duration are often found in participants with known risk factors (obesity) or pathologies (hypertension, diabetes); The goal of rehabilitation, therefore, requires providing much higher efforts. Therefore, the motivations for the practice of sport are essential and Deelen et al. (2018) notes in his study that health improvement goals (such as increasing energy level, stamina, or resistance to illness and disease) were the most prevalent goals for participation in sports.

Concerning blood pressure parameters, men's SBP decreased after 12 weeks of training. Reductions in blood pressure are generally reported in longitudinal studies on the benefits of physical and sports activities in hypertensive, normotensive, overweight, and normal-weight individuals (Brisebois et al., 2018; Whelton et al., 2002). A study of the longitudinal effects of "high-intensity functional training" showed a significant reduction in diastolic blood pressure (DBP) in men, while there was no change in the PAS in both men and women (Brisebois et al., 2018). However, a meta-study showed that the combination of aerobic and anaerobic exercises increased DBP more, while the SPB remained unchanged (Cornelissen and Smart, 2013). These conflicting results are also due to the nature of the protocol, their duration and the weekly frequency of the workouts.

Regarding cardiorespiratory fitness $\left(\mathrm{VO}_{2 \text { peak }}\right)$, there was no significant increase after 12 weeks of training, but the gains were more pronounced in men (7.5\%) as compared to women (5.4\%). Programs that lead to improvements in $\mathrm{VO}_{2 \text { peak }}$ incorporated exercises that require strong cardiovascular function and significant muscle mass such as CrossFit or HITF, with the advantage of reducing sitting time (20 to $60 \mathrm{~min}$ ) (Murawska-Cialowicz et al., 2015). Each session of this study included mixed exercises with a final duration of $1 \mathrm{hr}$ at a less intense pace.

In terms of heart rate, this study revealed a significant increase in HR1 and HRmax (maximum number of beats that a subject cannot exceed regardless of their level of training) in men and women after 3 months training session. These results are in line with those of Guirado et al. (2012) which reported the increase of HRmax after 6 months of training. The explanation is physiological because a workout is characterized by a progressive increase in the 
loads imposed on the subject. This allows each step to impose on the body solicitations close to the limits of its functional possibilities, hence the observation of an increase in the maximum heart rate.

The results of the survey on eating habits (number of meals per day) and alcohol consumption showed that the impact was not very significant on the parameters studied. However, some of our participants who consumed alcohol presented low $\mathrm{VO}_{\text {2peak }}$ values compared to those who did not or rarely consumed it.

This study, which assessed the benefits on some anthropometric and physiological parameters of a training program offered by a fitness center, revealed minor improvements which were highly depend on eating behaviors and lifestyle of participants after 3 months. Subsequent studies will evaluate the parameters studied after 12 weeks of training with a comparative approach of different centers.

\section{CONFLICT OF INTEREST}

No potential conflict of interest relevant to this article was reported.

\section{REFERENCES}

Brisebois MF, Rigby BR, Nichols DL. Physiological and fitness adaptations after eight weeks of high-intensity functional training in physically inactive adults. Sports (Basel) 2018;6:146.

Choukem SP, Kamdeu-Chedeu J, Leary SD, Mboue-Djieka Y, Nebongo DN, Akazong C, Mapoure YN, Hamilton-Shield JP, Gautier JF, Mbanya JC. Overweight and obesity in children aged 3-13 years in urban Cameroon: a cross-sectional study of prevalence and association with socio-economic status. BMC Obes 2017;4:7.

Cornelissen VA, Smart NA. Exercise training for blood pressure: a systematic review and meta-analysis. J Am Heart Assoc 2013;2:e004473.

Deelen I, Ettema D, Kamphuis CBM. Sports participation in sport clubs, gyms or public spaces: How users of different sports settings differ in their motivations, goals, and sports frequency. PLoS One 2018;13: e0205198.

Eime RM, Harvey JT, Charity MJ, Payne WR. Population levels of sport participation: implications for sport policy. BMC Public Health 2016; $16: 752$.

Guessogo WR, Mandengue SH, Assomo Ndemba PB, Medjo UO, Minye EE, Ahmaidi S, Temfemo A. Physical and functional follow-up of tuberculosis patients in initial intensive phase of treatment in Cameroon using the 6-min walk test. J Exerc Rehabil 2016a;12:333-339.
Guessogo WR, Temfemo A, Mandengue SH, Assomo Ndemba PB, Messina Ondoua RT, Hamadou A, Etoundi-Ngoa LS, Ahmaidi S. Effect of 24-week repeated short-time walking based training program on physical fitness of black Cameroonian obese women. J Exerc Rehabil 2016b; 12:90-98

Guirado GN, Damatto RL, Matsubara BB, Roscani MG, Fusco DR, Cicchetto LA, Seki MM, Teixeira AS, Valle AP, Okoshi K, Okoshi MP. Combined exercise training in asymptomatic elderly with controlled hypertension: effects on functional capacity and cardiac diastolic function. Med Sci Monit 2012;18:CR461-465.

Hug SM, Hartig T, Hansmann R, Seeland K, Hornung R. Restorative qualities of indoor and outdoor exercise settings as predictors of exercise frequency. Health Place 2009;15:971-980.

Juma PA, Mohamed SF, Matanje Mwagomba BL, Ndinda C, Mapa-Tassou C, Oluwasanu M, Oladepo O, Abiona O, Nkhata MJ, Wisdom JP, Mbanya JC. Non-communicable disease prevention policy process in five African countries. BMC Public Health 2018;18(Suppl 1):961.

Kamphuis CB, Van Lenthe FJ, Giskes K, Huisman M, Brug J, Mackenbach JP. Socioeconomic status, environmental and individual factors, and sports participation. Med Sci Sports Exerc 2008;40:71-81.

Kohl HW 3rd, Craig CL, Lambert EV, Inoue S, Alkandari JR, Leetongin G, Kahlmeier S; Lancet Physical Activity Series Working Group. The pandemic of physical inactivity: global action for public health. Lancet 2012;380:294-305.

Léger LA, Mercier D, Gadoury C, Lambert J. The multistage 20 metre shuttle run test for aerobic fitness. J Sports Sci 1988;6:93-101.

Murawska-Cialowicz E, Wojna J, Zuwala-Jagiello J. Crossfit training changes brain-derived neurotrophic factor and irisin levels at rest, after wingate and progressive tests, and improves aerobic capacity and body composition of young physically active men and women. J Physiol Pharmacol 2015;66:811-821.

Navti LK, Ferrari U, Tange E, Bechtold-Dalla Pozza S, Parhofer KG. Contribution of socioeconomic status, stature and birth weight to obesity in Sub-Saharan Africa: cross-sectional data from primary school-age children in Cameroon. BMC Public Health 2014;14:320.

Park HY, Jung WS, Kim J, Lim K. Twelve weeks of exercise modality in hypoxia enhances health-related function in obese older Korean men: a randomized controlled trial. Geriatr Gerontol Int 2019;19:311-316.

Posnakidis G, Aphamis G, Giannaki CD, Mougios V, Aristotelous P, Samoutis G, Bogdanis GC. High-intensity functional training improves cardiorespiratory fitness and neuromuscular performance without inflammation or muscle damage. J Strength Cond Res 2020 Feb 27 [Epub]. https://doi.org/10.1519/JSC.0000000000003516.

Schroeder EC, Welk GJ, Franke WD, Lee DC. Associations of health club membership with physical activity and cardiovascular health. PLoS 
One 2017;12:e0170471.

Sun S, Zhang H, Kong Z, Shi Q, Tong TK, Nie J. Twelve weeks of low volume sprint interval training improves cardio-metabolic health outcomes in overweight females. J Sports Sci 2019;37:1257-1264.

Whelton SP, Chin A, Xin X, He J. Effect of aerobic exercise on blood pressure: a meta-analysis of randomized, controlled trials. Ann Intern Med
2002;136:493-503.

Wong PC, Chia MY, Tsou IY, Wansaicheong GK, Tan B, Wang JC, Tan J, Kim CG, Boh G, Lim D. Effects of a 12-week exercise training programme on aerobic fitness, body composition, blood lipids and C-reactive protein in adolescents with obesity. Ann Acad Med Singapore 2008;37:286-293. 